\title{
Sarcopenia and Visceral Obesity are Significantly Related to Severe Storage Symptoms in Geriatric Female Patients
}

\author{
Mamoru Hashimoto (iD) \\ Nobutaka Shimizu (D) \\ Mitsuhisa Nishimoto' \\ Takafumi Minami ${ }^{1}$ \\ Kazutoshi Fujita' \\ Kazuhiro Yoshimura (iD) \\ Akihide Hirayama (D) ${ }^{2}$ \\ Hirotsugu Uemura' \\ 'Department of Urology, Kindai \\ University Faculty of Medicine, Osaka- \\ Sayama, Japan; ${ }^{2}$ Department of Urology, \\ Kindai Nara Hospital, Osaka-Sayama, \\ Japan
}

\begin{abstract}
Purpose: This study aimed to elucidate the relationship of psoas muscle atrophy and visceral obesity with lower urinary tract symptoms in geriatric female patients.

Patients and Methods: We retrospectively reviewed the medical records of female patients aged $\geq 65$ years. The psoas muscle index was defined, using computed tomography, as the cross-sectional area of the psoas muscle at the third lumbar vertebral level divided by the body surface area. We also measured visceral fat area at the umbilical level using computed tomography. We used logistic regression analysis to examine the relationships between the International Prostate Symptom Score (total score, voiding subscore, and storage subscore) and variables, such as age, body mass index, psoas muscle index, and visceral fat area. The International Prostate Symptom Score was categorized as mild, moderate, or severe.

Results: One hundred thirty-nine patients were included in our study. In the logistic regression analysis, we found statistically significant relationships between severe (versus mild-to-moderate) International Prostate Symptom Score storage subscore and variables, including low and high levels of psoas muscle index and visceral fat area, respectively. We could not find any significant relationships between the International Prostate Symptom Score total score and voiding subscore and the variables.
\end{abstract}

Conclusion: Psoas muscle atrophy and visceral fat accumulation are potential risk factors for severe storage symptoms in female patients aged $\geq 65$ years.

Keywords: female, International Prostate Symptom Score, overactive bladder symptom, psoas muscle, visceral fat

\section{Introduction}

In 1989, Irwin Rosenberg coined the term "sarcopenia", which meant age-related muscle volume loss, that rapidly aging societies were unprecedentedly facing. ${ }^{1}$ The cause of sarcopenia is broadly divided into two categories; primary sarcopenia (aging itself without any other cause) and secondary sarcopenia (eg, disuse, malnutrition, cachexia, and endocrine disorders), though they sometimes overlap. ${ }^{2}$ Patients with sarcopenia are forced to drastically change their lives, and their families are obliged to devote much of their time and energy in caring for these patients. A diagnosis code for sarcopenia was created in the tenth revision of the International Statistical Classification of Diseases and Related Health Problems in 2016; this diagnosis code addresses the condition as a disease itself, suggesting that sarcopenia should be treated and prevented. ${ }^{3}$
Correspondence: Nobutaka Shimizu Department of Urology, Kindai University Faculty of Medicine, 377-2, Ohno-Higashi, Osaka-Sayama, Osaka, 589-85 I I, Japan

Tel +8I-72-366-022I

Fax +8I-72-365-6273

Email shimizun@med.kindai.ac.jp 
The European Working Group on Sarcopenia in Older People proposed that sarcopenia is defined by low muscle mass and function. ${ }^{2}$ Muscle mass is not necessarily proportional to muscle function. ${ }^{4}$ The psoas muscle area (PMA), which reflects muscle mass, is obtained by computed tomography (CT) and has been proposed as a marker of sarcopenia. ${ }^{5}$ A pressure flow study in geriatric male patients by Majima et al concluded that PMA could have a significant relationship with impaired detrusor function. ${ }^{6}$ However, a similar examination using the CTbased evaluation of PMA in geriatric female patients is yet to be conducted. Further, the relationship of PMA with the lower urinary tract symptom (LUTS) questionnaires has never been investigated.

Women generally experience their final menstrual period at the median age of 51.4 years. Estrogen receptors (ERs) are present in the skeletal muscle, and the decrease in estrogen levels after menopause could lead to sarcopenia. ${ }^{7}$ Institutionalized geriatric people with functional difficulties develop detrusor hyperactivity with impaired contractility (DHIC). ${ }^{8}$ Visceral obesity also tends to be accelerated after menopause, and which could be associated with exacerbation of overactive bladder (OAB) symptoms. ${ }^{9,10}$ Hence, we hypothesized that PMA and visceral fat area (VFA), which were evaluated by $\mathrm{CT},{ }^{6,9}$ could have a significant relationship with LUTS, especially $\mathrm{OAB}$ symptoms, in geriatric female patients. Although the exact molecular mechanisms to support the hypothesis are obscure, these age-related changes could indirectly cause LUTS. Further, it has also been reported that DHIC is more pronounced in geriatric mice than younger ones and that age-related genomic bladder changes contribute to the development of DHIC. ${ }^{11}$ The prevalence of DHIC was found to be high in institutionalized geriatric people with functional difficulties; ${ }^{8}$ skeletal muscle atrophy caused by aging and disuse might have indirectly cause bladder dysfunctions in this population. Furthermore, long-term caloric restriction in aged rats reduced inflammatory and immune responses, while improving lower urinary tract functions. ${ }^{12}$ Thus, visceral fat accumulation might increase such responses and result in lower urinary tract dysfunctions.

In the previous Japanese epidemiological study, the most frequent female LUTS were storage symptoms like nocturia and a high daytime urination frequency, followed by weak stream, stress urinary incontinence, urinary urgency, and urge urinary incontinence. Furthermore, storage symptoms significantly affected quality of life. ${ }^{13}$
A previous report suggested that the incidence of $\mathrm{OAB}$ symptoms in women increases with an increase in their age. ${ }^{14}$ Thus, it was clinically important to elucidate the factors responsible for the development of LUTS, especially the storage symptoms. To our knowledge, the simultaneous influence of PMA and VFA on LUTS has never been investigated. Thus, we recruited female patients aged $\geq 65$ years and examined the impact of PMA and VFA on symptoms through a questionnaire.

\section{Materials and Methods}

We retrospectively analyzed female patients aged $\geq 65$ years who visited our department between 2008 and 2018. The ethics committee of the Faculty of Medicine, Kindai University, approved the study protocol (approval number: R02-106) and waived individual consent for this retrospective analysis. We preserve participants' anonymity and confidentiality. This study was conducted in accordance with the declaration of Helsinki. The CT-based cross-sectional area measurement of the PMA at the third lumbar vertebral level was conducted by manual tracing using Volume Analyzer SYNAPSE VINCENT (SYNAPSE 3D), FUJIFILM Corporation, Tokyo, Japan. The PMA was analyzed by a single investigator who was blind to all the demographic data (PMA is shown in Figure 1). Psoas muscle index (PMI) was defined as PMA divided by body surface area. ${ }^{6}$ VFA at the umbilical level was automatically measured on CT, with an attenuation range of -30 to -190 (VFA is shown in Figure 2). ${ }^{9}$ For clarity the original figures were modified from the one created by Volume Analyzer SYNAPSE VINCENT (SYNAPSE 3D). We performed CT scans using one of

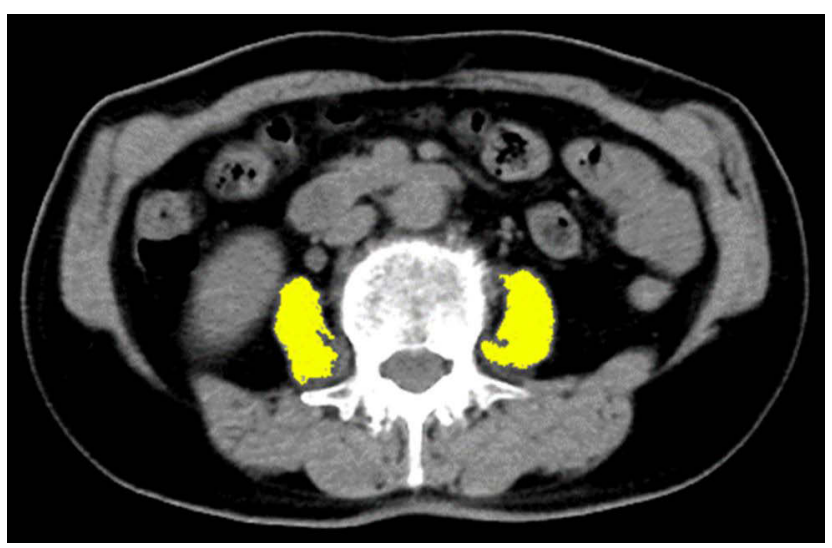

Figure I The cross-sectional area of the psoas muscle, termed psoas muscle area (PMA), at the third lumbar vertebral level on computed tomography (CT). PMA is measured by manual tracing, and PMI is calculated by divided the PMA by body surface area. PMA is in yellow. 


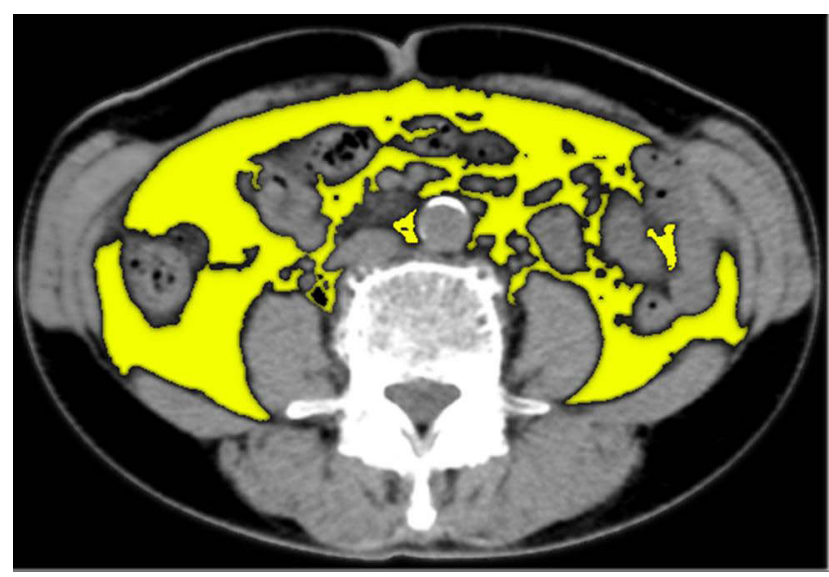

Figure 2 The cross-sectional area of the visceral fat area (VFA) at the umbilical level was automatically measured on CT. VFA is in yellow.

three types of multi-detector CT systems with 64 rows of detectors (TSX101/4A Aquilion PRIME, Light Speed Discovery 750HD, Light Speed VCT). We only included patients with a CT performed within 1 year before or after visiting our hospital and excluded patients with diabetes mellitus, neurological disease, acute cystitis, symptomatic urinary calculi, history of abdominal surgery, pelvic organ prolapse, interstitial cystitis, pelvic cancer, or urethral or ureteral catheterization as well as those on medication for LUTS.

We used the International Prostate Symptom Score (IPSS) for the evaluation of LUTS. The IPSS was categorized as follows according to a previous report: ${ }^{15}$ IPSS total score (IPSS-T) 0-7, 8-19, and 20-35; IPSS voiding subscore (IPSS-V) 0-6, 7-13, and 14-20; and IPSS storage subscore (IPSS-S) $0-5,6-9$, and $10-15$ as mild, moderate and severe, respectively. The IPSS was validated by a symptom questionnaire for the assessment of female LUTS. $^{16}$

Statistical analyses were performed using EZR (Saitama Medical Center, Jichi Medical University, Saitama, Japan), which is a graphical user interface for $\mathrm{R}$ (The R Foundation for Statistical Computing, Vienna, Austria). ${ }^{17}$ Logistic regression analyses were performed to examine the association of each variable, including PMI, VFA, body mass index (BMI), and age, with IPSS (severe vs mild-to-moderate). Mann-Whitney $U$-test was performed to compare the differences in variables between groups, which were categorized according to mild, moderate, and severe IPSS. P $<0.05$ was considered statistically significant. Scattered plots with empty circles showing distributions of values were created in
GraphPad Prism version 8.00 for Windows, GraphPad Software, La Jolla California USA, www.graphpad.com.

\section{Results}

After adopting the exclusion and inclusion criteria, we enrolled 139 female patients aged $\geq 65$ years. Table 1 shows the patients' characteristics. Previous reports found that dyslipidemia is proportional to visceral fat volume and that some antihypertensive drugs could affect LUTS. ${ }^{9,18,19}$ Furthermore, patients with malignancies could face a risk of developing sarcopenia. ${ }^{2}$ Thus, we also disclosed the prior history of malignancies and the use of antihypertensive and antihyperlipidemic drugs in Table 1. Tables 2 and 3 demonstrate that IPSS-T and IPSS-V had no significant association with any variables in the multivariate regression analysis. In contrast, Table 4 shows that statistically significant relationships were found between severe IPSS-S and low and high levels of PMI [odds ratio (OR) (95\% confidence interval (CI)): 1.03

Table I Patients' Characteristics $(n=139)$

\begin{tabular}{|c|c|}
\hline \multicolumn{2}{|l|}{ Data are Median (Range), or $\mathrm{n}(\%)$} \\
\hline Age (year) & $74(65,98)$ \\
\hline Height (m) & $151(136,165)$ \\
\hline Weight (kg) & $51(31,93)$ \\
\hline BMI $\left(\mathrm{kg} / \mathrm{m}^{2}\right)$ & $22.0(13.7,38.7)$ \\
\hline $\operatorname{PMA}\left(\mathrm{m}^{2}\right)$ & $8.6(4.7,16.6)$ \\
\hline PMI $\left(\mathrm{m}^{2} / \mathrm{m}^{2}\right)$ & $5.94(2.82,11.1)$ \\
\hline VFA $\left(\mathrm{cm}^{2}\right)$ & $83.2(9.23,209.2)$ \\
\hline IPSS-T & $6(0,35)$ \\
\hline IPSS-V & $2(0,20)$ \\
\hline IPSS-S & $4(0,15)$ \\
\hline \multicolumn{2}{|l|}{ Antihypertensive Drug } \\
\hline$\alpha$-blocker & $6(4 \%)$ \\
\hline Calcium channel blocker & $39(28 \%)$ \\
\hline ACE-I or ARB & $35(25 \%)$ \\
\hline Diuretic & $16(12 \%)$ \\
\hline Antihyperlipidemic drug & $30(22 \%)$ \\
\hline \multicolumn{2}{|l|}{ Prior Cancer History } \\
\hline Renal cell carcinoma & $12(9 \%)$ \\
\hline Breast cancer & II (8\%) \\
\hline Lung cancer & $4(3 \%)$ \\
\hline Hematologic cancer & $4(3 \%)$ \\
\hline Gastric cancer & $2(1 \%)$ \\
\hline Adrenal cancer & $\mathrm{I}(\mathrm{I} \%)$ \\
\hline Renal pelvis and ureter cancer & I (I\%) \\
\hline
\end{tabular}

Abbreviations: $\mathrm{BMI}$, body mass index; PMA, psoas muscle area; PMI, psoas muscle index; VFA, visceral fat area; IPSS, International Prostate Symptom Score; IPSS-T, IPSS total score; IPSS-V, IPSS voiding subscore; IPSS-S, IPSS storage subscore; ACE-I, angiotensin-converting enzyme inhibitor; ARB, angiotensin receptor blocker. 
Table 2 The Association of IPSS-T (Mild 0-7 and Moderate 8-19 versus Severe 20-35) with Variables Was Analyzed Using Multivariate Logistic Regression Analysis

\begin{tabular}{|l|l|l|l|l|}
\hline \multirow{2}{*}{} & \multicolumn{2}{|l|}{ Univariate Analysis } & \multicolumn{2}{l|}{ Multivariate Analysis } \\
\cline { 2 - 5 } & OR $(95 \% \mathrm{Cl})$ & p-value & $\begin{array}{l}\text { OR } \\
(95 \% \mathbf{C l})\end{array}$ & p-value \\
\hline Age (year) & $1.01(0.92$ to $1.1 \mathrm{l})$ & 0.81 & & \\
BMI $\left(\mathrm{kg} / \mathrm{m}^{2}\right)$ & $0.98(0.83$ to 1.15$)$ & 0.78 & & \\
PMI $\left(\mathrm{m}^{2} / \mathrm{m}^{2}\right)$ & $0.85(0.54$ to 1.34$)$ & 0.47 & & \\
VFA $\left(\mathrm{cm}^{2}\right)$ & $0.99(0.98$ to 1.01$)$ & 0.84 & & \\
\hline
\end{tabular}

Abbreviations: IPSS-T, International Prostate Symptom Score total score; OR, odds ratio; $\mathrm{Cl}$, confidence interval; $\mathrm{BMI}$, body mass index; PMI, psoas muscle index; VFA, visceral fat area.

Table 3 The Association of IPSS-V (Mild 0-6 and Moderate 7-13 versus Severe 14-20) with Variables Was Analyzed Using Multivariate Logistic Regression Analysis

\begin{tabular}{|l|l|l|l|l|}
\hline \multirow{2}{*}{} & \multicolumn{2}{|l|}{ Univariate Analysis } & \multicolumn{2}{l|}{ Multivariate Analysis } \\
\cline { 2 - 5 } & OR (95\% Cl) & p-value & $\begin{array}{l}\text { OR } \\
(95 \% ~ C l)\end{array}$ & p-value \\
\hline Age (year) & $0.99(0.89$ to $1.1 \mathrm{l})$ & 0.92 & & \\
BMI $\left(\mathrm{kg} / \mathrm{m}^{2}\right)$ & $1.05(0.90$ to 1.24$)$ & 0.53 & & \\
PMI $\left(\mathrm{m}^{2} / \mathrm{m}^{2}\right)$ & $0.86(0.51$ to 1.44$)$ & 0.56 & & \\
VFA $\left(\mathrm{cm}^{2}\right)$ & $0.99(0.98$ to 1.01$)$ & 0.41 & & \\
\hline
\end{tabular}

Abbreviations: IPSS-V, International Prostate Symptom Score voiding subscore; $\mathrm{OR}$, odds ratio; $\mathrm{Cl}$, confidence interval; $\mathrm{BMI}$, body mass index; PMI, psoas muscle index; VFA, visceral fat area.

Table 4 The Association of IPSS-S (Mild 0-5 and Moderate 6-9 versus Severe 10-15) with Variables Was Analyzed Using Multivariate Logistic Regression Analysis

\begin{tabular}{|l|l|l|l|l|}
\hline \multirow{2}{*}{} & \multicolumn{2}{|l|}{ Univariate Analysis } & \multicolumn{2}{l|}{ Multivariate Analysis } \\
\cline { 2 - 5 } & OR $(95 \% \mathrm{Cl})$ & p-value & OR $(95 \% \mathrm{Cl})$ & p-value \\
\hline Age (year) & $1.03(0.96$ to $\mathrm{I} .10)$ & 0.48 & & \\
BMI $\left(\mathrm{kg} / \mathrm{m}^{2}\right)$ & $1.09(0.98$ to 1.22$)$ & 0.12 & & \\
PMI $\left(\mathrm{m}^{2} / \mathrm{m}^{2}\right)$ & $0.66(0.45$ to 0.97$)$ & $0.03^{*}$ & $0.62(0.40$ to 0.96$)$ & $0.03^{*}$ \\
VFA $\left(\mathrm{cm}^{2}\right)$ & $1.02(\mathrm{I} .01$ to $\mathrm{l} .03)$ & $0.002^{* *}$ & $1.02(1.00$ to 1.03$)$ & $0.003^{*}$ \\
\hline
\end{tabular}

Abbreviations: IPSS-S, International Prostate Symptom Score storage subscore; $\mathrm{OR}$, odds ratio; $\mathrm{Cl}$, confidence interval; $\mathrm{BMI}$, body mass index; PMI, psoas muscle index; VFA, visceral fat area.

(0.96 to 1.10$), \mathrm{p}$-value $=0.03]$ and VFA $[\mathrm{OR}(95 \% \mathrm{CI})$ : $1.02(1.00$ to 1.03$), \mathrm{p}$-value $=0.003]$, respectively (Table 4). However, BMI was not considered as a significant predictor of severe IPSS-S. PMI and VFA levels of individuals with severe IPSS-S were significantly different from those of individuals in the mild and moderate IPSS-S groups (Figures 3 and 4, respectively). Thus, psoas muscle atrophy and visceral obesity were significantly related to severe storage symptoms.

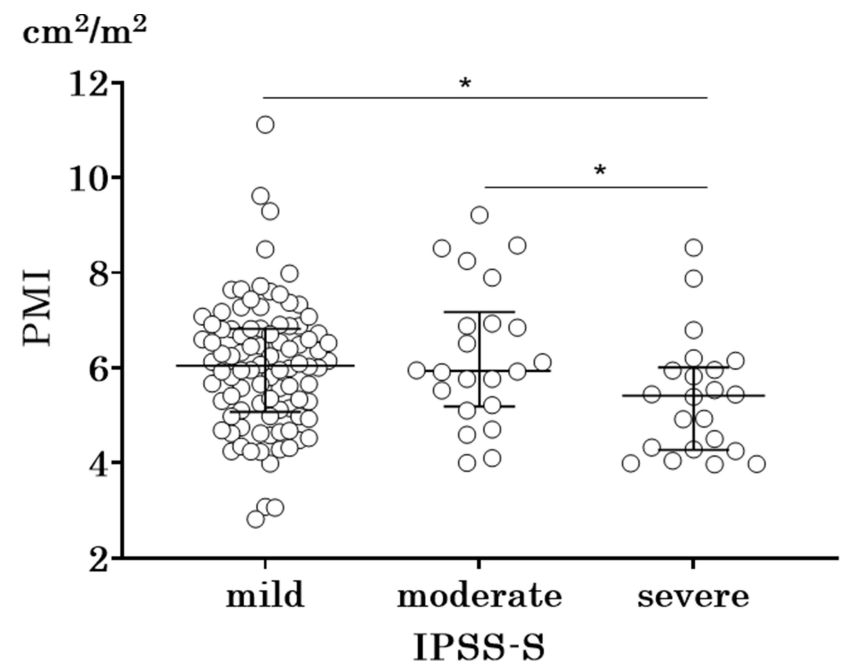

Figure 3 The horizontal lines represent the median and interquartile ranges. Scatter plots with empty circles represent the distributions of each value in three groups, which are categorized as follows: IPSS-S mild 0-5, moderate 6-9, and severe 10-15. Mann-Whitney U-test was performed to compare differences in PMI among the three groups. Statistical significance was considered as follows: $* \mathrm{P}<0.05, * * \mathrm{P}<0.01$.

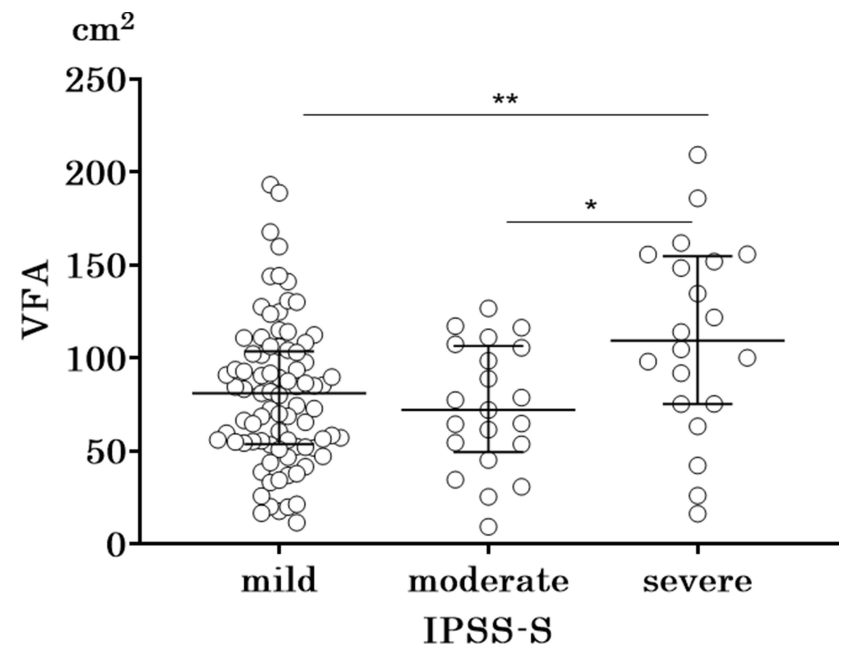

Figure 4 The horizontal lines represent the median and interquartile ranges. Scatter plots with empty circles represent the distributions of each value in three groups, which are categorized as follows: IPSS-S mild 0-5, moderate 6-9, and severe 10-15. Mann-Whitney U-test was performed to compare differences in VFA among the three groups. Statistical significance was considered as follows: $* \mathrm{P}<0.05$, **P $<0.01$.

\section{Discussion}

This study demonstrated that low PMI and high VFA levels have a significant relationship with severe IPSS-S in elderly female patients aged $\geq 65$ years. A previous report stated that low skeletal muscle mass was a potential diagnostic marker for sarcopenia in men and women. ${ }^{5}$ Moreover, previous reports have suggested that geriatric females with sarcopenia have detrusor overactivity (DO) or DHIC. ${ }^{8,20}$ Thus, low levels of PMI could have 
significant detrimental influences on storage symptoms in geriatric female patients. Meanwhile, subcutaneous fat tends to be redistributed to the visceral area in geriatric females. Visceral fat accumulation is more correlated with systemic metabolic disorders than other variables like subcutaneous fat and BMI. ${ }^{7,9}$ A previous report suggested that there was a significant correlation between metabolic syndrome and OAB symptoms. ${ }^{10}$ The $\beta 3$-adrenoceptor ( $\beta 3-\mathrm{AR}$ ) functions by governing lipolytic actions in omental adipose tissues and is especially dominant among the noradrenaline receptors in obese individuals. The stimulation of $\beta 3$-AR results in free fatty acid release from abundant omental adipose tissue into the circulatory system in cases of visceral obesity, which causes systemic metabolic disorders. Additionally, Trp64Arg is a $\beta 3$-AR gene in which mutation and polymorphism are reported to induce the development of visceral obesity and $\mathrm{OAB}$, respectively. ${ }^{9,21}$ Therefore, high levels of VFA might be associated with severe IPSS-S.

Women generally have their final menstrual period at the median age of 51.4 years. Skeletal muscles with ERs can maintain muscle volume and function through estrogen signaling, but menopause gradually exacerbates its homeostasis over time. ${ }^{7}$ Therefore, sarcopenia could be induced after menopause in a time-dependent manner and is reportedly associated with DHIC. ${ }^{8}$ ERs are also present in the pelvic organ, bladder, and vagina. ${ }^{22,23}$ Estrogen exhaustion results in atrophy and fibrosis of these organs after menopause, leading to the exacerbation of OAB symptoms. ${ }^{24,25}$ In terms of obesity, estrogen prevents visceral fat accumulation, whereas it maintains subcutaneous fat depots. Thus, visceral fat accumulation tends to be accelerated after menopause, and aging also contributes to it. ${ }^{9}$ According to these previous reports, skeletal muscle volume loss, visceral obesity, and $\mathrm{OAB}$ might progress on exposure to estrogen shortages for longer periods. Local estrogen administration, rather than systemic therapies, is a promising treatment option for postmenopausal women with $\mathrm{OAB}$ symptoms. ${ }^{25}$

Our results strongly suggest that preventing sarcopenia at a young age might reduce the chance of severe storage symptom development at an old age in women. Despite previous pharmaceutical trials for the treatment and prevention of sarcopenia, no definitive pharmacological treatments have been marketed. ${ }^{26,27}$ To date, exercise is the foundation for sarcopenia prevention; however, high quality evidence derived from randomized controlled trials using unified diagnostic criteria is required. ${ }^{28}$ Generally, exercise strengthens the topical muscle; however, it is worth noting that the skeletal muscle is a secretory organ. Cytokines called myokines are released from the muscle itself after physical activity, causing systemic effects on several organs, including the adipose tissue, brain, liver, pancreas, and immune system. ${ }^{29}$ Although evidence of crosstalk between the skeletal muscle and bladder mediated by myokines remains unavailable, an ovariectomized rat experiment has reported that exercise preserves bladder function and morphology. ${ }^{30}$ Furthermore, it is reported that exercise reduces visceral fat volume. ${ }^{9}$ A urodynamic study of women reported that obesity was significantly associated with higher abdominal pressure. ${ }^{31}$ Higher abdominal pressure induced by visceral fat accumulation might compromise bladder capacity. In previous research, long-term caloric restriction in aged rats reduced inflammatory and immune responses and contributed to the prevention of lower urinary tract dysfunctions. ${ }^{12}$ Thus, visceral fat accumulation could result in storage symptom deterioration through reduced bladder capacity and exacerbated inflammatory and immune responses. A previous study suggested that increased levels of adipokines and C-reactive protein (CRP) were observed in OAB patients. ${ }^{32}$ Furthermore, inflammatory cytokines produced by visceral fat accumulation are involved in the progression of sarcopenia. IL-6 and CRP correlated with fat mass could be predictors of future muscle loss. Leptin, another substance produced by fat, contributes to the suppression of obesity; however, its effect might decrease after significant visceral fat accumulation. Fat deposition in muscle is increased secondary to leptin resistance, which results in poor muscle quality. There is a high possibility that obesity will progress because of physical inactivity. ${ }^{33}$ A previous article indicated that leptin is anti-cholinergic. It reduces $\mathrm{Ca}^{2+}$ influx in smooth muscle, decreases the synthesis of acetylcholine receptors, and ameliorates detrusor hyperactivity. Although this hypothesis needs further analysis, we believe that the resistance of leptin might exacerbate $\mathrm{OAB}$ symptoms. ${ }^{34}$ In summary, muscle loss and visceral fat accumulation synergistically get worse and could worsen storage symptoms.

Our study, however, is not without limitations. First, estrogen shortages after menopause could influence not only muscle volume but also LUTS. It was possible that deteriorating storage symptoms were directly caused by estrogen shortages. However, we could not evaluate estrogen levels in this retrospective study. Second, we evaluated PMA at the third lumbar vertebral level using CT. 
Unfortunately, we could not assess the impact of muscle function on LUTS. Third, whether the prevention of sarcopenia and obesity reduce the development of severe storage symptoms in geriatric females should be evaluated in a long-term randomized controlled trial.

\section{Conclusion}

Psoas muscle atrophy and visceral obesity resulted in severe storage symptoms in women aged $\geq 65$ years. Further investigation is necessary to clarify whether aginginduced skeletal muscle atrophy might have detrimental influences on lower urinary tract functions and whether exercise could reverse it. Moreover, the impact of obesity, especially visceral fat accumulation on storage symptoms, should be examined in future studies.

\section{Disclosure}

The authors report no conflicts of interest in this work.

\section{References}

1. Rosenberg H. Summary comments. Am J Clin Nutr. 1989;50:1231S1233S. doi:10.1093/ajen/50.5.1231

2. Cruz-Jentoft AJ, Baeyens JP, Bauer JM, et al. Sarcopenia: European consensus on definition and diagnosis: report of the European working group on sarcopenia in older people. Age Ageing. 2010;39 (4):412-423. doi:10.1093/ageing/afq034

3. Anker SD, Morley JE, von Haehling S. Welcome to the ICD-10 code for sarcopenia. J Cachexia Sarcopenia Muscle. 2016;7(5):512-514. doi:10.1002/jcsm.12147

4. Goodpaster BH, Park SW, Harris TB, et al. The loss of skeletal muscle strength, mass, and quality in older adults: the health, aging and body composition study. J Gerontol A Biol Sci Med Sci. 2006;61 (10):1059-1064. doi:10.1093/gerona/61.10.1059

5. Hamaguchi Y, Kaido T, Okumura S, et al. Proposal for new diagnostic criteria for low skeletal muscle mass based on computed tomography imaging in Asian adults. Nutrition. 2016;32(1112):1200-1205. doi:10.1016/j.nut.2016.04.003

6. Majima T, Funahashi Y, Matsukawa Y, et al. Investigation of the relationship between bladder function and sarcopenia using pressure flow studies in elderly male patients. Neurourol Urodyn. 2019;38 (5):1417-1422. doi:10.1002/nau.24001

7. Messier V, Rabasa-Lhoret R, Barbat-Artigas S, Elisha B, Karelis AD, Aubertin-Leheudre M. Menopause and sarcopenia: a potential role for sex hormones. Maturitas. 2011;68(4):331-336. doi:10.1016/j. maturitas.2011.01.014

8. Resnick NM, Yalla SV. Detrusor hyperactivity with impaired contractile function. An unrecognized but common cause of incontinence in elderly patients. JAMA. 1987;257(22):3076-3081. doi:10.1001/ jama.1987.03390220074024

9. Wajchenberg BL. Subcutaneous and visceral adipose tissue: their relation to the metabolic syndrome. Endocr Rev. 2000;21(6): 697-738.

10. Bunn F, Kirby M, Pinkney E, et al. Is there a link between overactive bladder and the metabolic syndrome in women? A systematic review of observational studies. Int J Clin Pract. 2015;69(2):199-217. doi:10.1111/ijcp.12518
11. Kamei J, Ito H, Aizawa N, et al. Age-related changes in function and gene expression of the male and female mouse bladder. Sci Rep. 2018;8(1):2089. doi:10.1038/s41598-018-20406-0

12. Ito H, Kamei J, Aizawa N, et al. Preventive effects of long-term caloric restriction on aging related in vivo bladder dysfunction and molecular biological changes in the bladder and dorsal root ganglia in rats. J Urol. 2016;196(5):1575-1583. doi:10.1016/j.juro.2016.05.104

13. Takahashi S, Takei M, Asakura H. Clinical guidelines for female lower urinary tract symptoms (second edition). Int $J$ Urol. 2021;28:474-492. doi:10.1111/iju.14492

14. Homma Y, Yamaguchi O, Hayashi K. An epidemiological survey of overactive bladder symptoms in Japan. BJU Int. 2005;96 (9):1314-1318. doi:10.1111/j.1464-410X.2005.05835.x

15. Gacci M, Sebastianelli A, Salvi M, et al. Central obesity is predictive of persistent storage lower urinary tract symptoms (LUTS) after surgery for benign prostatic enlargement: results of a multicentre prospective study. BJU Int. 2015;116(2):271-277. doi:10.1111/ bju. 13038

16. Hsiao SM, Lin HH, Kuo HC. International Prostate Symptom Score for assessing lower urinary tract dysfunction in women. Int Urogynecol J. 2013;24(2):263-267. doi:10.1007/s00192-0121818-8

17. Kanda Y. Investigation of the freely available easy-to-use software 'EZR' for medical statistics. Bone Marrow Transplant. 2013;48 (3):452-458. doi:10.1038/bmt.2012.244

18. Salman M, Khan AH, Sulaiman SAS, et al. Effect of calcium channel blockers on lower urinary tract symptoms: a systematic review. Biomed Res Int. 2017;2017:4269875. doi:10.1155/2017/4269875

19. Kawamoto B, Shimizu S, Shimizu T, et al. Angiotensin II centrally induces frequent detrusor contractility of the bladder by acting on brain angiotensin II type 1 receptors in rats. Sci Rep. 2016;6:22213. doi:10.1038/srep22213

20. Valentini FA, Robain G, Marti BG. Urodynamics in women from menopause to oldest age: What motive? What diagnosis? Int Braz J Urol. 2011;37(1):100-107. doi:10.1590/S1677-55382011000100 013

21. Qu HC, Zhang W, Liu YL, Wang P. Association between polymorphism of $\beta 3$-adrenoceptor gene and overactive bladder: a meta-analysis. Genet Mol Res. 2015;14(1):2495-2501. doi:10.4238/2015.March. 30.7

22. Tincello DG, Taylor AH, Spurling SM, Bell SC. Receptor isoforms that mediate estrogen and progestagen action in the female lower urinary tract. J Urol. 2009;181(3):1474-1482. doi:10.1016/j.juro.20 08.10 .104

23. Blakeman PJ, Hilton P, Bulmer JN. Oestrogen and progesterone receptor expression in the female lower urinary tract, with reference to oestrogen status. BJU Int. 2000;86(1):32-38. doi:10.1046/j.1464410x.2000.00724.x

24. Domoney C. Treatment of vaginal atrophy. Womens Health. 2014;10 (2):191-200. doi:10.2217/WHE.14.9

25. Cardozo L, Robinson D. Special considerations in premenopausal and postmenopausal women with symptoms of overactive bladder. Urology. 2002;60(5Suppl 1):64-71. doi:10.1016/S0090-4295(02)017 99-5

26. Dhillon RJ, Hasni S. Pathogenesis and management of sarcopenia. Clin Geriatr Med. 2017;33(1):17-26. doi:10.1016/j.cger.2016.08.002

27. Kwak JY, Kwon KS. Pharmacological interventions for treatment of sarcopenia: current status of drug development for sarcopenia. Ann Geriatr Med Res. 2019;23:98-104. doi:10.4235/agmr.19.0028

28. Yoshimura Y, Wakabayashi H, Yamada M, Kim H, Harada A, Arai H. Interventions for treating sarcopenia: a systematic review and meta-analysis of randomized controlled studies. $J$ Am Med Dir Assoc. 2017;18(6):553.e1-553.e16. doi:10.1016/j.jamda.2017.03.019

29. Pedersen BK, Febbraio MA. Muscles, exercise and obesity: skeletal muscle as a secretory organ. Nat Rev Endocrinol. 2012;8(8):457-465. doi:10.1038/nrendo.2012.49 
30. Magaldi FM, Moreno M, Magaldi CM, et al. Resistance exercise evokes changes on urinary bladder function and morphology in hypoestrogen rats. Front Physiol. 2020;29(10):1605. doi:10.3389/fphys.2019.01605

31. Richter HE, Creasman JM, Myers DL, et al. Urodynamic characterization of obese women with urinary incontinence undergoing a weight loss program. Int Urogynecol J Pelvic Floor Dysfunct. 2008;19(12):1653-1658. doi:10.1007/s00192-008-0694-8

32. Liu HT, Jiang YH, Kuo HC. Increased serum adipokines implicate chronic inflammation in the pathogenesis of overactive bladder syndrome refractory to antimuscarinic therapy. PLoS One. 2013;8(10): e76706. doi:10.1371/journal.pone.0076706
33. Zamboni M, Mazzali G, Fantin F, Rossi A, Di Francesco V. Sarcopenic obesity: a new category of obesity in the elderly. Nutr Metab Cardiovasc Dis. 2008;18(5):388-395. doi:10.1016/j.numecd. 2007.10.002

34. Wuntakal R, Sharma S, Kaler M, Hollingworth T. Leptin - a novel pharmacological agent for treatment of women with overactive bladder symptoms? Med Hypotheses. 2012;79(6):856-857. doi:10.1016/j. mehy.2012.09.006

\section{Publish your work in this journal}

Research and Reports in Urology is an international, peer-reviewed, open access journal publishing original research, reports, editorials, reviews and commentaries on all aspects of adult and pediatric urology in the clinic and laboratory including the following topics Pathology, pathophysiology of urological disease; Investigation and treatment of urological disease; Pharmacology of drugs used for the treatment of urological disease. The manuscript management system is completely online and includes a very quick and fair peer-review system, which is all easy to use. Visit http://www.dovepress.com/ testimonials.php to read real quotes from published authors. 